\title{
The Role of Serum Levels of Insulin-Like Growth Factor-1 and Brain-Derived Neurotrophic Factor in Predicting Recovery in Stroke Samah Bastawy ${ }^{1, *}$, Ali Mahmoud Ahmed ${ }^{2}$ \\ ${ }^{1}$ Department of Clinical Pathology, ${ }^{2}$ Department of Neurology, Faculty of Medicine, Helwan University, Cairo, Egypt \\ *Correspondence to Samah Bastawy, Email: bastawysamah@yahoo.com
}

\begin{abstract}
Background: Despite the neurotrophins like brain-derived neurotrophic factor and insulin-like growth factor-1 are thought to have important role in neurorehabilitation, the factors that affect these biomarkers are not well known.

Objectives: We aimed to investigate the effect of maximal aerobic exercise on serum neurotrophins levels in patients with chronic stroke and to investigate the factors that affect their resting and exercise-associated levels.

Patients and Methods: Potential factors of resting and exercise-related serum neurotrophins levels were studied among 35 patients with chronic stroke. Resting brain derived neurotrophic factor, resting insulin-like growth factor, exercisedependent change brain derived neurotrophic factor, and exercise- dependent change in insulin-like growth factor were studied using demographic, disease burden, and cardiometabolic factors.

Results: Brain-derived neurotrophic factor did not change after exercise $(p=0.38)$ while insulin-like growth factor decreased $(\mathrm{p}=0.001)$. Higher resting brain-derived neurotrophic factor was determined by greater lower extremity impairment $\left(\mathrm{p}=0.004, \mathrm{r}^{2}=0.23\right)$. The greater response of brain-derived neurotrophic factor to exercise was determined by higher fluid intelligence $\left(\mathrm{p}=0.01, \mathrm{r}^{2}=0.18\right)$. Resting insulin-like growth factor-1 was not changed by all of the studied factors. Conclusion: Resting and percent change in brain-derived neurotrophic factor were associated with cognitive and physical recovery after chronic stroke. On the other hands, insulin-like growth factor-1 was not useful in chronic stroke. Interestingly, fluid intelligence was positively associated with exercise-related rise in brain-derived neurotrophic factor.
\end{abstract}

Keywords: Neurotrophins; IGF-1; BDNF; Stroke.

\section{INTRODUCTION}

The use of established biomarkers is a part of personalized medicine as it aims to guide the proper medical treatment ${ }^{(\mathbf{1})}$. Despite stroke biomarkers, like neuroimaging and genetic testing, have been established (2), serum levels of neurotrophins (NTs) are relatively lowcost biomarkers requiring minimal health-care resources (e.g. blood sample). Furthermore, NTs, like brain-derived neurotrophic factor (BDNF) and insulin-like growth factor-1 (IGF-1) are proposed to be important factors for assessing neurorehabilitation in stroke.

IGF-1 is an auspicious biomarker for stroke as the low resting levels of serum IGF-1 is correlated with bad outcome. Supportingly, Åberg et al. ${ }^{(3)}$ demonstrated acute increase in the serum IGF-1(1-10 days) and three months after admission and predicted good outcome for up to two years (as defined by Modified Rankin Scale) after the stroke. Furthermore, Denti et al. ${ }^{(4)}$ revealed that patients with stroke and had low levels of IGF-1 were associated with worse outcome. Increased ratios of serum IGF-1 to bound IGF-1 on admission were proposed to predict good. National Institutes of Health Stroke Scale- (NIHSS) defined functional outcome at three months. Among 15 patients with acute stroke, patients with increased circulating IGF-1 had good predicted level of fitness before the stroke. However, there was a debate regarding whether increased or decreased levels of IGF-1 are correlated with good outcomes. In some studies, the measured serum levels of IGF-1 on admission did not show any association with functional outcome ${ }^{(5)}$.

Levels of serum BDNF and its function as a predictive biomarker is not well established. Previous study in chronic stroke has revealed that serum level of BDNF was not correlated with NIHSS-defined functional outcome and the levels declined in three months ${ }^{(6)}$. However, another study suggested that serum level of BDNF was stable up to six months after the stroke and did not relate to time since the occurrence of the stroke ${ }^{(7)}$. Stanne et al. ${ }^{(8)}$ demonstrated that serum BDNF level $<10$ days after the stroke was lower in patients with stroke than healthy patients and that decreased levels were correlated with bad functional outcome two years later. On the other hand, Santos et al. ${ }^{(7)}$ revealed that BDNF did not have association with motor function. Moreover, there is focus regarding BDNF as a biomarker of psychological outcomes since lower levels of BDNF has been correlated with the presence of depression after the stroke. Moreover, levels of BDNF may be related to BDNF genotype ${ }^{\left({ }^{9}\right)}$. Therefore, although NTs have potential predictive value for indicating recovery after stroke, their predictive value in stroke is not well-established.

NTs have been investigated at both rest and after biological stress. They have been revealed to rise,

Received:18/2/2019 
ejhm.journals.ekb.eg

decrease, or remain without changes after a bout of exercise ${ }^{(\mathbf{1 0})}$. Therefore, exercise associated NTs biomarkers are not well defined. Previous systematic review and meta-analysis showed that circulating BDNF increased after moderate to high-level exercise in healthy people. The intensity of exercise could determine the level of NTs. Recent study revealed that serum BDNF rose after moderate exercise in chronic stroke ${ }^{(\mathbf{1 1})}$. Intensity seems to be important in terms of the reaction of BDNF to exercise. However, its levels had not been studied after intense exercise in patients with stroke.

Although many studies were conducted to examine NTs as biomarkers in stroke recovery, it is not obvious whether resting or exercise-dependent NTs are appropriate biomarkers to guide the optimum treatment in chronic stroke. The causes were; low number of studied predictive variables ${ }^{(6,7)}$, the shortness of study period ( $\leq 6$ months post-stroke) ${ }^{(6,7)}$ and absence of NTs collection after exercise ${ }^{(8)}$. These studies had not defined the factor associated resting NTs levels in patients with chronic stroke. To address this, we investigated a comprehensive set of factors, demographic, physical and cognitive, that may explain the association of resting and exercisedependent serum NTs with chronic stroke after a bout of maximal aerobic exercise. We hypothesized that serum level of BDNF would rise but IGF-1 would fall after an acute bout of maximal exercise. We did not hypothesize predictor factors explaining resting or percent change of serum levels of NTs given equivocal reports ${ }^{(6,7)}$ and lack of studies explaining a range of predictive factors.

\section{MATERIALS AND METHODS}

Participants

This study included 35 stroke patients ( 23 males and 12 females) who had a stroke more than 6 months before the study and were eligible for safe participation (PAR-Q) (12) in an incremental maximal aerobic exercise. Written informed consent was obtained from all patients as well as approval of the Ethical Committee. This study was conducted under the Declaration of Helsinki.

\section{Blood collection and exercise protocol}

Samples of blood $(5 \mathrm{ml})$ were collected from the median cubital vein of patients before and after the conduction of an incremental maximal aerobic exercise test. Samples were collected within $7 \mathrm{~min}$ of the end of exercise to know the short duration bioavailability of exercise-dependent NTs ${ }^{(13)}$. Subsequently, blood samples were left for clotting for $30 \mathrm{~min}$, then centrifuged at $1500 \mathrm{~g}$ for $10 \mathrm{~min}$ at $20^{\circ} \mathrm{C}$ and then stored at $-80^{\circ} \mathrm{C}$. Serum IGF-1 and BDNF levels were determined using enzyme-linked immunosorbent assay (ELISA) using DuoSet human kits (R \& D Systems Inc. Minneapolis, $\mathrm{MN}, \mathrm{USA}$ ) according to the manufacturer's protocol. The exercise that was performed was in the form of incremental maximal aerobic exercise test and was performed on either a supported treadmill or on a total body recumbent stepper. $\dot{\mathrm{O}}_{2 \max }$ was defined as when participants reached a respiratory exchange ratio greater than 1.0 or within $10 \%$ of age-correlated maximum heart rate ${ }^{(14)}$.

\section{Outcome variables}

The levels of serum NTs at rest and after exercise were used to determine the percent change $(\Delta)$ of IGF-1 and BDNF. The assessed variables were: resting IGF-1, resting BDNF, $\triangle \mathrm{IGF}-1$, and $\triangle \mathrm{BDNF}$. Unlike IGF-1, we illustrated that the resting BDNF levels were correlated with the height $(\mathrm{m}, \mathrm{r} 2=0.461, \mathrm{p}=0.005)$. Therefore, we corrected the levels of BDNF by height, and that corrected values were used for all statistical analyses.

\section{Predictors of NTs variables}

We categorized potential predictors that can affect the serum levels of NTs into; demographic, disease burden, and cardiometabolic. The demographic variables were measured before the test and included: age, sex, weight, and height. Disease burdens included measuring of stroke severity (NIHSS), cognition [Montreal Cognitive Assessment (MoCA)], self-selected walking velocity $(\mathrm{cm} / \mathrm{s})$, measured on an instrumented walkway (Prokinetics, Havertown, PA, USA), fluid intelligence (Raven's Progressive Matrices), depression [Hospital Anxiety and Depression Scale (HADS)] and lower limb affection (Chedoke McMaster Stages of Recovery, CMSR). The cardiometabolic variables included: VO2max obtained during an incremental maximal aerobic exercise test, habitual levels of physical activity, and number of cardiometabolic comorbidities (15). Because IGF-1 and BDNFs are associated with insulin and glucose metabolism, respectively, diabetes condition as a distinct binary variable was included in the analyses. Self-reported duration, frequency, and type of physical activity in the last week were determined to calculate the metabolic equivalent of task (MET). We calculated MET-min/week for each activity using the Compendium of Physical Activities which correlates physical activities to METs.

All these predictor variables were visually examined by a histogram and tested for skewness using Shapiro-Wilk. Those variables showed non-normal distributions when dividing into disease-relevant categories. If disease categories did not reveal normal distribution, statistical distributions were used. MoCA was divided into either abnormal $(<26)$ or normal $(\geq 26)$. NIHSS was categorized into either minor $(<4)$ or moderate to severe effect $(>4){ }^{(16)}$. Minimum physical 
activity guidelines suggest approximately 675 MET-min per week (150 min of moderate intensity exercise per week) ${ }^{(14)}$. Physical activity levels categorized respondents in tertiles $(<786,786-1578$, and $>1578$ MET-min per week).

\section{Data analysis}

The paired sample $\mathrm{t}$ test was implemented to compare the levels of serum BDNF and IGF-1 at rest and after the incremental maximal aerobic exercise test with Cohen's $d$ that was determined to evaluate the level of diff erences between serum NTs. Means of RER, $\dot{\mathrm{VO}} 2_{\max }$, and maximum heart rate were determined from the test. Pearson correlation between NIHSS and $\dot{\mathrm{V} O} 2 \mathrm{max}$ was calculated to determine whether stroke-related impairment was correlated with $\dot{\mathrm{VO}} 2_{\max }$. The analysis was performed using the IBM SPSS statistical software version 24 (IBM Corporation, NY, USA). Four separate models for each outcome and predictors were performed. First, each explanatory variable was entered into a bivariate regression with each outcome variable. The predictor variables that were not considered predictive $(p>0.20)$ were removed. Subsequently, significant predictors were entered into stepwise linear regression models, one for each prementioned outcome variable. During this stepwise regression, predictors with $p$ values $<0.20$ were excluded one by one. After model of significant terms was obtained, removed predictors were re-added one-by-one.

\section{RESULTS}

\section{Patients characteristics and the test of incremental maximal aerobic exercise}

Patients' characteristics are summarized in table (1). Stroke severity and lower limb impairment indicated that our stroke patients had a moderate to severe impairment. The duration of the incremental maximal aerobic exercise test was $12 \mathrm{~min}$ and $46 \mathrm{~s} \pm 6 \mathrm{~min}$ and $4 \mathrm{~s}$ and the mean of $\dot{\mathrm{VO}} 2_{\text {max }}$ was $17.0 \pm 4.66 \mathrm{ml} \mathrm{O} 2 / \mathrm{min} / \mathrm{kg}$, which showed a negative correlation with stroke severity (NIHSS) $(\mathrm{p}=$

a

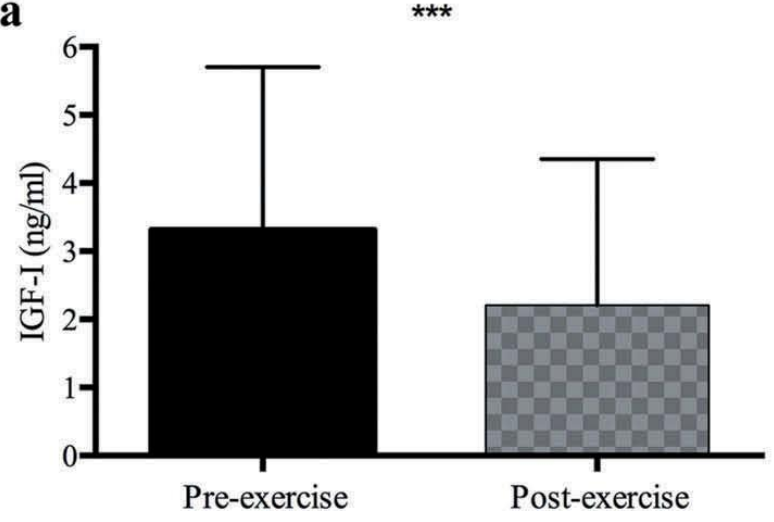

$\left.0.06, \mathrm{r}^{2}=-0.32\right)$. The number of patients who completed the test on a recumbent stepper was 13, while the number of patients who completed the test on a treadmill was 22 . The mean of RER was $1.01 \pm 0.089$ and patients completed the test at $99.5 \% \pm 21.3 \%$ of the maximum heart rate of the same age. Regarding $\dot{\mathrm{VO}} 2_{\max }, \triangle \mathrm{BDNF}$, and RER, there was no difference between patients performing maximal tests on treadmill compared to recumbent stepper $(\mathrm{p}=0.12, \mathrm{p}=0.78, \mathrm{p}=0.21$, respectively).

Table (1): The characteristics of patients

\begin{tabular}{|c|c|}
\hline Demographic & Patients $(\mathrm{N}=3 \mathrm{H}$ \\
\hline Age (years) & $65.2 \pm 9.4$ \\
\hline Height $(\mathrm{m})$ & $1.71 \pm 0.1$ \\
\hline Months since stroke & $31.5 \pm 26.7$ \\
\hline Stroke Severity (NIHSS) ${ }^{\mathrm{a}}$ & $4.29 \pm 4.4$ \\
\hline Lower limb impairment ${ }^{\mathrm{b}}$ & $10.4 \pm 3.3$ \\
\hline Cognitive function $^{\mathrm{c}}(\mathrm{MoCA})$ & $23.5 \pm 6.5$ \\
\hline Fluid intelligence (Raven's Matrices) & $23.3 \pm 7.2$ \\
\hline Haemorrhagic stroke & 9 \\
\hline Ischemic stroke & 26 \\
\hline \multicolumn{2}{|c|}{ 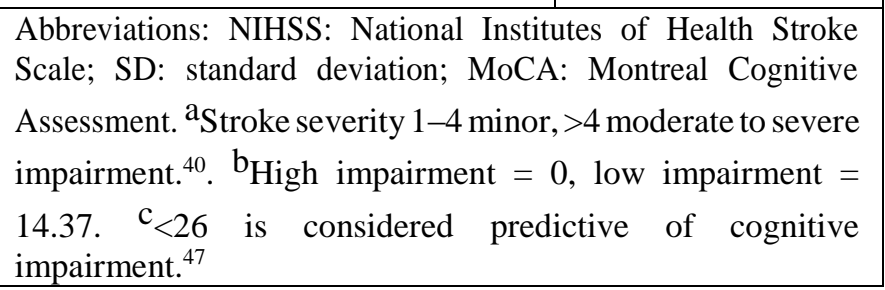 } \\
\hline
\end{tabular}

\section{Levels of IGF-1 and BDNF after exercise}

There was a significant decrease in the IGF-1 levels after exercise (mean difference $=0.98 \mathrm{ng} / \mathrm{ml}, \mathrm{p}=0.001$; Figure 1a). On the other hand, there was no difference regarding BDNF levels after exercise $(26.1 \pm 14.4$ vs. $28.1 \pm 15.0 \mathrm{ng} / \mathrm{ml} / \mathrm{m}, \mathrm{p}=0.383$; Figure $1 \mathrm{~b}$ ). The levels of BDNF before correction for height were $45.3 \pm 25.5$ vs. $48.3 \pm 26.7 \mathrm{ng} / \mathrm{ml})$.

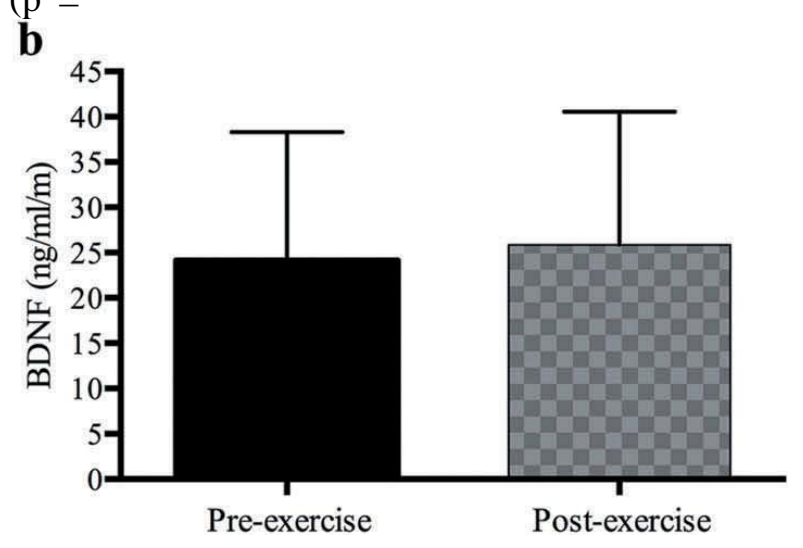

Figure (1): Serum IGF-1 (A) but not serum BDNF (B) levels decreased after graded maximal exercise test. 
ejhm.journals.ekb.eg

\section{Predicting variables for resting IGF-1 or $\triangle I G F-1$}

Having the resting IGF-1, bivariate regression indicated that diabetes diagnosis predicted higher levels of resting IGF-1. However, it was not retained in the next stepwise model $\left(\mathrm{p}=0.10, \mathrm{r}^{2}=0.28\right)$. Regarding $\triangle \mathrm{IGF}-1$, the bivariate regression analysis indicated that male gender $(\mathrm{p}=0.199, \mathrm{r} 2=0.22)$ and diabetes diagnosis $(\mathrm{p}=$ $\left.0.15, \mathrm{r}^{2}=0.25\right)$ predicted higher $\Delta \mathrm{IGF}-1$. However, it was not significant in the final stepwise linear regression model.

\section{Lower limb impairment and resting $B D N F$}

Bivariate regression analysis showed that younger age, lower physical activity (METs per week), greater stroke severity (NIHSS), and more severe lower-limb impairment (CMSR) $(\mathrm{p}=0.17, \mathrm{p}=0.09, \mathrm{p}=0.009, \mathrm{p}=$ 0.004 , respectively) predicted higher resting BDNF. In the last stepwise regression model, higher resting BDNF was predicted by more severe lower limb impairment $(\mathrm{T}$ (34) $=-3.80, p=0.001, r 2=0.31$; Figure 2) but not by age, stroke severity, or physical activity $(\mathrm{p}=0.46, \mathrm{p}=$ 0.60 , or $\mathrm{p}=0.58$; respectively).

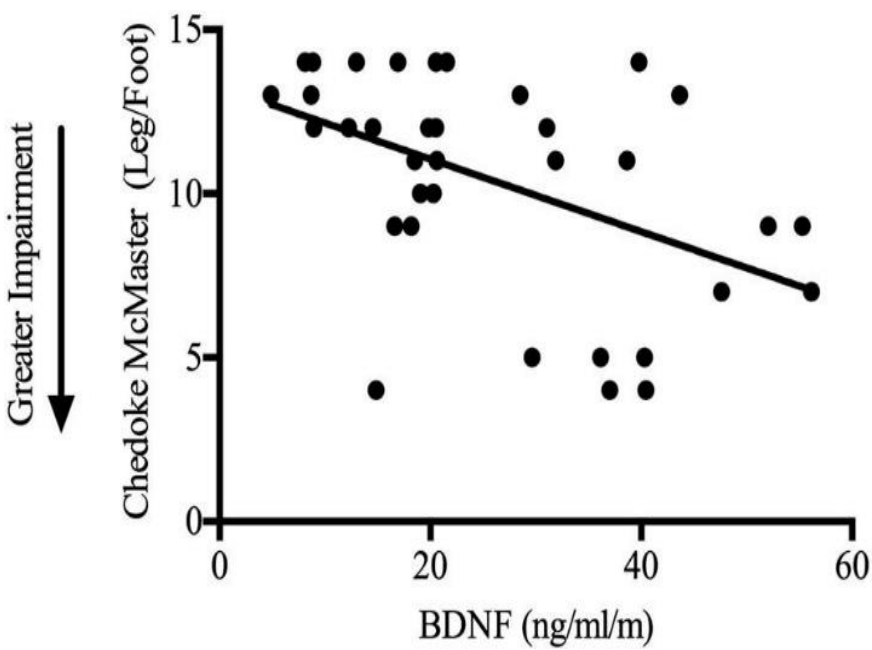

Figure (2): Greater lower limb impairment predicted higher resting serum BDNF.

\section{Fluid intelligence and $\triangle B D N F$ after exercise}

Bivariate regression indicated that greater levels of physical activity $(\mathrm{p}=0.04, \mathrm{r} 2=0.35)$ and higher fluid intelligence score $(\mathrm{p}=0.01, \mathrm{r} 2=0.43)$ predicted higher $\triangle B D N F$. In the last stepwise regression model, increase in BDNF after exercise was predicted by better fluid intelligence $(\mathrm{T}(34)=2.72, \mathrm{p}=0.01$, standardized $\beta=$ $0.43, \mathrm{r} 2=0.18$; Figure 3 , ) but not by physical activity ( $\mathrm{p}$ $=0.26$ ).

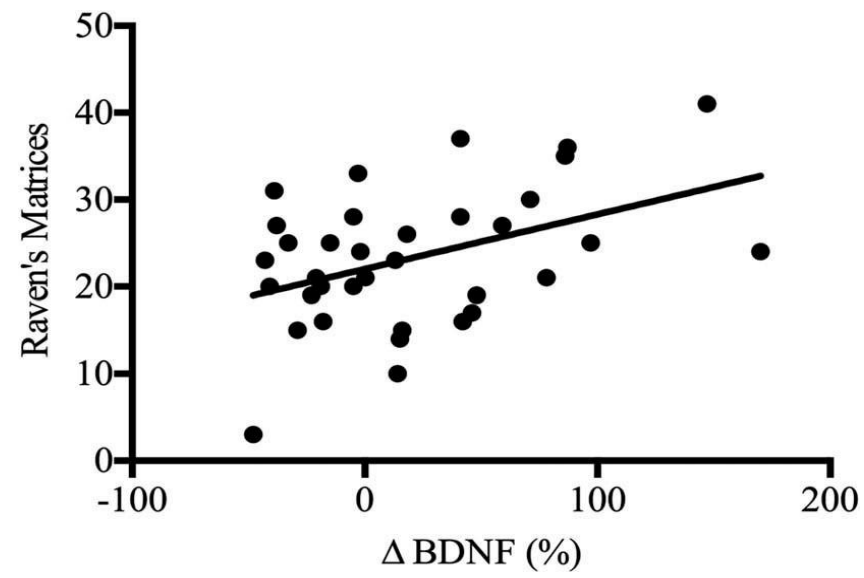

Figure (3): Better fluid intelligence predicted higher $\triangle \mathrm{BDNF}$ after maximal exercise.

\section{Model residual normality}

The Shapiro-Wilk test revealed that model residuals were distributed normally for resting BDNF and $\triangle \mathrm{BDNF}$ ( $p=0.16$ and 0.11 , respectively). However, the residuals for IGF-1 models were not calculated because there were no significant predictors in the final stepwise model.

\section{DISCUSSION}

We have illustrated through our study that IGF-1 decreased, but BDNF did not change after an incremental maximal aerobic exercise test in patients with stroke. Furthermore, greater limb impairment predicted higher levels of resting BDNF and fluid intelligence significantly predicted the increase in BDNF after exercise.

Our study revealed that after an incremental maximal aerobic exercise test, serum IGF-1 decreased among patients with chronic stroke. Previous literature revealed that post-exercise IGF-1 levels rose in elderly patients, declined in athletes, or revealed no change in adult males. During rest, IGF-1 is released mainly from the liver ${ }^{(\mathbf{1 7})}$; however, after stress or exercise, IGF-1 is thought to be transiently released in muscle or endothelial cells by proteolytic cleavage of IGFBP ${ }^{(\mathbf{1 8 - 2 1 )}}$. A previous study in rodent models showed that, after exercise, IGF-1 passed through the blood brain barrier (BBB) to ease neurogenesis and expression of IGF-1 after exercise ${ }^{(22)}$. Trejo et al. ${ }^{(23)}$ showed that exerciseinduced hippocampal and cerebellar neurogenesis was hindered by an IGF-1 binding factor. We hypothesized that exercise-induced decreases in IGF-1 might reflect the absorption across the BBB. However, IGF-1 has generalized physiological actions and therefore it is important to put into consideration that IGF-1 might be decreased after exercise for many reasons ${ }^{(24)}$. One possible reason is IGF-1's function in glucose 
metabolism. it has been demonstrated to increase glucose uptake and glycolysis and therefore, it is possible that serum IGF-1 declined after onset of exercise to control energy metabolism.

Having the BDNF, recent meta-analyses revealed that both resistance and aerobic exercise moderately increased BDNF in healthy people (Hedge's $\mathrm{G}=0.46$ ) (15). However, exercise-induced elevation requires enough intensity and duration. In patients with chronic stroke, de Morais et al. (11) revealed that moderate exercise increased BDNF. Our observation that the resistance of serum BDNF to exercise may reflect our patients' reduced physical capacity to exercise at enough intensity and duration. Our population demonstrated fitness levels lower than what is regarded as enough to carry out activities of daily living ${ }^{(25,26)}$. In fact, the mean duration of the incremental maximal aerobic exercise test was shorter than other exercise protocols shown to increase BDNF ${ }^{(\mathbf{1 5})}$.

Our results showed that resting serum IGF-1 and BDNF were $20-35$ times lower ${ }^{(27)}$ and 5-14 times higher than previously published studies in other chronic stroke patients, respectively. It seems that the low levels of aerobic fitness and moderate to severe levels of disability are the two factors that contributes to these findings. In normal adults, lower $\dot{\mathrm{VO}} 2_{\max }$ is associated with decreased levels of resting IGF-1 and increased levels of $\mathrm{BDNF}^{(28)}$. Despite we revealed no association between IGF-1 and fitness, Mattlage et al. ${ }^{(29)}$ revealed that estimated $\mathrm{VO} 2$ in patients with acute stroke was associated with lower levels of IGF-1. Our sample had lower levels of aerobic fitness and IGF-1 than that of the the patients reported by Mattlage et al. ${ }^{(29)}$.

Having the disability, previous study by Rodier $\boldsymbol{e} t$ al. ${ }^{(6)}$ revealed that resting serum BDNF levels after stroke by one day was highest when stroke severity scores were highest. Moreover, resting levels of BDNF after stroke by 1.5 months decreased with stroke severity. Therefore, it seems that severe lower limb impairment is associated with greater serum BDNF that may explain the levels we observed in our study. In chronic stroke, not only central nervous system damage occurs but also atrophy of peripheral tissue that leads to further impairment. BDNF is expressed in peripheral sensory nerves and vascular endothelial cells, stored in platelets, and released after peripheral injury. Thus, we hypothesized that the increased levels of BDNF in our study could be a result of peripheral tissue injury or waste associated with hemiparesis.

Fluid intelligence is defined as the ability of problem-solving and reasoning. It is particularly important to perform correctly in demanding environments like stroke rehabilitation. BDNF is hypothesized to have low BBB permeability; therefore, the volume of infarction and functional recovery in rodents are improved when BDNF BBB transport is exhibited ${ }^{(30)}$. However, there is a recent suggestion that the BBB may be disrupted in chronic stroke and selectively permeable after exercise. Therefore, exercise might provide a unique opportunity for BDNF-mediated rehabilitation in patients with chronic stroke. Moreover, irisin is released into circulation during stress or exercise from muscle and pass the BBB to facilitate BDNF expression. Therefore, the levels of BDNF in brain could also be increased through irisin which is released during exercise.

In rats, exercise increases BDNF in brain regions important for cognition, like the prefrontal cortex ${ }^{(31)}$. Therefore, reduced BDNF in the frontal and parietal cortex of human is associated with poor cognition like Alzheimer's disease ${ }^{(31)}$. In fact, these two areas are thought to be important for fluid intelligence ${ }^{(32)}$. Our study associated better fluid intelligence with an increase in serum BDNF in response to exercise and put into consideration BDNF as a potential cognitive biomarker in patients with chronic stroke. Rather than Raven's Matrices scores, other factors have roles. Therefore, future studies are required to elucidate the association between fluid intelligence and the levels of BDNF related to exercise.

\section{CONCLUSION}

In conclusion, our study examined whether functional and cognitive factors in patients with chronic stroke predict exercise-related changes in NTs. There were weak relationships with resting and exercisedependent BDNF not with IGF-1. These findings illustrate that serum BDNF and IGF-1 might not be highly indicative biomarkers for chronic stroke. However, our study illustrated a novel finding that the change in serum BDNF, after maximal exercise, in patients with chronic stroke was positively associated with fluid intelligence.

\section{REFERENCES}

1. Bierman AS, Tinetti ME (2016): Precision medicine to precision care: managing multimorbidity. Lancet, 388 (10061): 2721-2723.

2. Stinear CM (2017): Prediction of motor recovery after stroke: advances in biomarkers. Lancet Neurol., 16 (10): 826836.

3. Åberg $\mathrm{D}$, Jood $\mathrm{K}$, Blomstrand $\mathrm{C}$, Jern $\mathrm{C}$, Nilsson $\mathrm{M}$, Isgaard J, Å berg ND (2011): Serum IGF-I levels correlate to improvement of functional outcome after ischemic stroke. J Clin Endocrinol Metab., 96 (7): E1055-E1064.

4. Denti L, Annoni V, Cattadori E, Salvagnini MA, Visioli S, Merli MF et al. (2004): Insulin-like growth factor 1 as a 
predictor of ischemic stroke outcome in the elderly. Am $\mathbf{J}$ Med., 117 (5): 312-317.

5. Okazaki H, Beppu H, Mizutani K, Okamoto S, Sonoda S (2014): Changes in serum growth factors in stroke rehabilitation patients and their relation to hemiparesis improvement. J Stroke Cerebrovascular, 23 (6): 1703-1708.

6. Rodier M, Quirié A, Prigent-Tessier A, Béjot Y, Jacquin A, Mossiat C et al. (2015): Relevance of post-stroke circulating BDNF levels as a prognostic biomarker of stroke outcome. Impact of rt-PA treatment. PloS one, 10 (10): e0140668.

7. Santos GL, Alcântara CC, Silva-Couto MA, GarcíaSalazar LF, Russo TL (2016): Decreased brain-derived neurotrophic factor serum concentrations in chronic poststroke subjects. J Stroke Cerebrovascular Dis., 25 (12): 29682974.

8. Stanne TM, Åberg ND, Nilsson S, Jood K, Blomstrand C, Andreasson $U$ et al. (2016): Low circulating acute brainderived neurotrophic factor levels are associated with poor long-term functional out- come after ischemic stroke. Stroke, 47 (7): 1943-1945.

9. Mang CS, McEwen LM, MacIsaac JL, Snow NJ, Campbell KL, Kobor MS et al. (2017): Exploring genetic influences underlying acute aerobic exercise eff ects on motor learning. Sci Rep-Uk., 7 (1): 12123.

10. Stokes K, Nevill M, Frystyk J, Lakomy H, Hall G (2005): Human growth hormone responses to repeated bouts of sprint exer- cise with diff erent recovery periods between bouts. J Appl Physiol., 99 (4): 1254-1261.

11. Morais VA, Tourino MF, Almeida AC, Albuquerque TB, Linhares RC, Christo PP et al. (2017): A single session of moderate intensity walking increases brain-derived neurotrophic factor (BDNF) in the chronic post-stroke patients. Top Stroke Rehabil., 25 (1): 1-5.

12. Thomas S, Reading J, Shephard RJ (1992): Revision of the physical activity readiness questionnaire (PAR-Q). Can J Sport Sci., 17 (4): 338-345.

13. Price RD, Milne SA, Sharkey J, Matsuoka N (2007): Advances in small molecules promoting neurotrophic function. Pharmacol Ther., 115 (2): 292-306.

14. Ferguson B (2014): ACSM's guidelines for exercise testing and prescription 9th Ed. 2014. J Can Chiropr Assoc., 58 (3): 328.

15. Szuhany KL, Bugatti M, Otto MW (2015): A meta-analytic review of the eff ects of exercise on brain-derived neurotrophic factor. J Psychiatr Res., 60: 56-64.

16. Khatri P, Kleindorfer DO, Yeatts SD, Saver JL, Levine SR, Lyden PD et al. (2010): Strokes with minor symptoms: an exploratory analysis of the National Institute of Neurological Disorders and Stroke recombinant tissue plasminogen activator trials. Stroke, 41 (11): 2581-2586.

17. Yakar S, Liu JL, Stannard B, Butler A, Accili D, Sauer B et al. (1999): Normal growth and development in the absence of hepatic insulin-like growth factor I. Proc Nat Acad Sci., 96 (13): 7324-7329.

18. Berg U, Gustafsson T, Sundberg CJ, Kaijser L, CarlssonSkwirut C, Bang P (2007): Interstitial IGF-I in exercising skeletal muscle in women. Eur J Endocrinol., 157 (4): 427435.

19. Berg U, Bang P (2004): Exercise and circulating insulin-like growth factor I. Hormone Res Paediatrics, 62 (1): 50-58.

20. Pedersen BK, Febbraio MA (2012): Muscles, exercise and obesity: skeletal muscle as a secretory organ. Nat Rev Endocrinol., 8 (8): 457.

21. Booth BA, Boes M, Dake BL, Knudtson KL, Bar RS (2002): IGFBP-3 binding to endothelial cells inhibits plasmin and thrombin proteolysis. Am J Physiol Endocrinol Metab., 282 (1): E52-E58.

22. Carro E, Trejo JL, Busiguina S, Torres-Aleman I (2001): Circulating insulin-like growth factor I mediates the protective eff ects of physical exercise against brain insults of diff erent etiology and anatomy. J Neurosci., 21 (15): 56785684

23. Trejo JL, Carro E, Torres-Aleman I (2001): Circulating insulin-like growth factor I mediates exercise-induced increases in the number of new neurons in the adult hippocampus. J Neurosci., 21 (5): 1628-1634.

24. Puche JE, Castilla-Cortázar I (2012): Human conditions of insulin-like growth factor-I (IGF-I) deficiency. J Transl Med., 10 (1): 224.

25. Arnett SW, Laity JH, Agrawal SK, Cress ME (2008): Aerobic reserve and physical functional performance in older adults. Age Ageing, 37 (4): 384-389.

26. Ploughman M, Kelly LP (2016): Four birds with one stone? Reparative, neuroplastic, cardiorespiratory, and metabolic benefits of aerobic exercise poststroke. Curr Opin Neurol., 29 (6): $684-692$.

27. Silva-Couto MD, Prado-Medeiros CL, Oliveira AB, Alcântara CC, Guimaraes AT, Salvini TD et al. (2014): Muscle atrophy, voluntary activation distur- bances, and low serum concentrations of IGF-1 and IGFBP-3 are associated with weakness in people with chronic stroke. Phys Ther., 94 (7): 957-967.

28. Hwang J, Castelli DM, Gonzalez-Lima F (2017): The positive cognitive impact of aerobic fitness is associated with peripheral inflammatory and brain-derived neurotrophic biomarkers in young adults. Physiol Behav., 179: 75-89.

29. Mattlage AE, Rippee MA, Abraham MG, Sandt J, Billinger SA (2017): Estimated prestroke peak vo2 is related to circulating igf-1 levels during acute stroke. Neurorehabil Neural Repair, 31 (1): 65-71.

30. Fujimura H, Altar CA, Chen R, Nakamura T, Nakahashi T, Kambayashi JI et al. (2002): Brain-derived neurotrophic factor is stored in human platelets and released by agonist stimulation. Thromb Haemost., 88 (04): 728-734.

31. Uysal N, Kiray M, Sisman AR, Camsari UM, Gencoglu C, Baykara B et al. (2015): Eff ects of volun- tary and involuntary exercise on cognitive functions, and VEGF and BDNF levels in adolescent rats. Biotech Histochem., 90 (1): 55-68.

32. Au J, Sheehan E, Tsai N, Duncan GJ, Buschkuehl M, Jaeggi SM (2015): Improving fluid intelli- gence with training on working memory: a meta-analysis. Psychon B Rev., 22 (2): 366-377. 